\title{
miR-29b in regulating blood pressure and cardiac function in the rat model of hypertension
}

\author{
XIUJIANG HAN* ${ }^{*}$ CHAO WANG ${ }^{*}$, YONGJIAN LI, ZHE JIN, BOYA ZHANG and YANG DONG \\ Department of Cardiology, Tianjin Hospital of ITCWM, Nankai Hospital, Nankai, Tianjin 300100, P.R. China
}

Received July 27, 2018; Accepted January 18, 2019

DOI: $10.3892 / \mathrm{etm} .2019 .7335$

\begin{abstract}
The possibility of micro ribonucleic acid-29b (miR-29b) regulating blood pressure and cardiac function in the rat model of hypertension was investigated. Sixty rat models of hypertension were established and randomly divided into the lentivirus group $(n=20)$, the negative lentivirus group $(n=20)$ and the control group $(n=20)$. Rats in the lentivirus group were injected with the recombinant lentivirus, and those in the negative lentivirus and control groups were injected with the negative control virus and infection enhancement solution, respectively. The systolic pressure of rats was monitored using the tail-cuff method, and changes in the cardiac function of rats were evaluated via high-frequency ultrasound. At 3 weeks after virus infection, rats were weighed and sacrificed, the heart was taken and the left ventricular mass index was calculated. Moreover, the expression of miR-29b in myocardial tissues was detected via reverse transcription-quantitative polymerase chain reaction (RT-qPCR). The systolic pressure in the lentivirus group was significantly decreased compared with those in the negative lentivirus and control groups $(\mathrm{P}<0.05)$. In the lentivirus group, the systolic pressure was significantly reduced after virus transfection $(\mathrm{P}<0.05)$, and there were also statistically significant differences in ultrasonic measurement indexes (LVPWT, IVST, LVEDD and LVESD) $(\mathrm{P}<0.05)$. LVPWT was remarkably decreased at 5 weeks and 6 weeks compared with that in the previous week, and it was lower than those in the other two groups (all $\mathrm{P}<0.05$ ). After virus transfection, IVST in the lentivirus group showed a decreasing trend, which was obviously lower than those in the other two groups $(\mathrm{P}<0.05)$. After virus transfection, LVEDD in the lentivirus group increased gradually, and was higher than that in the other two groups. The expression of miR-29b was upregulated in the lentivirus group compared with those in the other two
\end{abstract}

Correspondence to: Dr Xiujiang Han, Department of Cardiology, Tianjin Hospital of ITCWM, Nankai Hospital, 6 Changjiang Road, Nankai, Tianjin 300100, P.R. China

E-mail:xf67mb@163.com; superloongwang@163.com

*Contributed equally

Key words: miR-29b, hypertension, rat model, blood pressure, cardiac function groups $(\mathrm{P}<0.05)$. The overexpression of miR-29b can reduce the blood pressure and significantly improve the cardiac function of hypertension rats.

\section{Introduction}

Hypertension refers to a chronic disease with increased diastolic and/or systolic pressure, which endangers kidneys, heart, brain and other organs and leads to organic damage through injuring blood vessels, thereby threatening organ function and life $(1,2)$. With the changes in people's living habits and the increase in psychological pressure, the incidence rate of hypertension has also been increasing each year $(3,4)$. Hypertension is a long-term chronic disease, but hypertension-induced myocardial infarction/cerebral infarction, heart failure/renal failure and other target organ damage have extremely high disability and fatality rates, placing great psychological and economic burdens on individuals and families (5). The heart is one of the important organs directly damaged by hypertension, and long-term cardiovascular hypertension leads to vascular remodeling, followed by gradual cardiac remodeling due to response to such chronic volume overload pressure, in which the left ventricular hypertrophy is the most significant. Such persistent remodeling will result in arrhythmia, and severe heart failure will cause sudden death $(6,7)$. More than $30 \%$ of hypertension patients have ventricular remodeling in clinic. Therefore, it is necessary to investigate the possibility of regulating cardiac function in hypertension and prevent heart failure and sudden death in hypertension patients.

Micro ribonucleic acid (miRNAs) is a kind of endogenous non-coding inhibitor, which can bind to the target RNA through complementary base pairing to interfere in the function of target RNA and have an inhibitory effect on various types of tumors $(8,9)$. A number of studies have demonstrated that the miR-29 family has a close correlation with fibrosis, and it was found that miR-29 can inhibit fibrosis in the heart and lungs (10). Widlansky et al (11) found in the rat model of diabetes mellitus type II that the miR-29 family is necessary for the endothelial function in normal human and animal models, which also has great therapeutic potential for cardiac metabolic disturbance. Luo et al (12) found in the mouse experiment that the downregulated miR-29b can break the elastin, increase the collagen deposition inside the blood vessel and also increase the degree of thoracic aortic stiffness, thus leading to hypertension (13). In the present study, the 
Table I. Preparation of reaction system.

\begin{tabular}{lllr}
\hline Reverse transcription & & \multicolumn{2}{c}{ PCR } \\
\hline $2.5 \mathrm{U} / \mu 1$ Poly A polymerase & $1 \mu \mathrm{l}$ & miR-29b primer (10 $\mu \mathrm{M})$ & $2 \mu 1$ \\
Rtase mixture & $1 \mu \mathrm{l}$ & Universal Adaptor PCR Primer $(2 \mu \mathrm{M})$ & $2 \mu 1$ \\
$5 \mathrm{X}$ reaction buffer & $5 \mu \mathrm{l}$ & 2X All-in-One qPCR Mix & $10 \mu 1$ \\
Total RNA & $2 \mu \mathrm{g}$ & cDNA & $2 \mu 1$ \\
Add RNase free $\mathrm{H}_{2} \mathrm{O}$ to $25 \mu \mathrm{l}$ & & Add RNase free $\mathrm{H}_{2} \mathrm{O}$ to $20 \mu \mathrm{l}$ & \\
\hline
\end{tabular}

possibility of miR-29b in regulating blood pressure and cardiac function in the rat model of hypertension was investigated, so as to provide a new therapeutic target for hypertension patients and a potential marker for diagnosing cardiac damage in hypertension.

\section{Materials and methods}

Laboratory animals. A total of 60 male specific pathogen-free rats with spontaneous hypertension aged 3 months and with a weight of 260-290 g were purchased and fed for 1 week to adapt to the laboratory environment $\left(24^{\circ} \mathrm{C}, 12 / 12\right.$ light/dark cycles and humidity $60 \pm 10 \%$ ) with free access to water and food. The systolic pressure of rats in quiet and waking conditions were measured via caudal artery using the non-invasive blood pressure measurement and analysis system (tail-cuff method) at about 10 a.m. after the tail was heated for $5 \mathrm{~min}$. It was measured every $5 \mathrm{~min}$ for 2 weeks until the blood pressure became $150 \mathrm{mmHg}$. After feeding for 3 weeks, rats were randomly divided into the lentivirus group $(n=20)$, the negative lentivirus group $(n=20)$ and the control group $(n=20)$.

The study was approved by the Ethics Committee of Tianjin Hospital of ITCWM, Nankai Hospital (Nankai, China).

Reagents and materials. ZH-HX-Z non-invasive blood pressure measurement and analysis system for spontaneously hypertensive rats (Anhui Zhenghua Biological Instrument Equipment Co., Ltd., Huaibei, China), lentivirus with miR-29b overexpression sequence and negative control virus (Shanghai Zhonghong Boyuan Biological Technology Co., Ltd., Shanghai, China), high-efficiency lentivirus transfection enhancement solution (Shanghai Umibio Science and Technology Co., Ltd., Shanghai, China), ultrasound diagnostic instrument (Henan Enpusi Electronic Technology Co., Ltd., Henan, China), $10 \%$ chloral hydrate (Shanghai Jianglai Biotechnology Co., Ltd., Shanghai, China), TRIzol reagent, chloroform and isopropanol (Thermo Fisher Scientific, Inc., Shanghai, China), reverse transcription reagent and $2 \mathrm{X}$ All-in-One miRNA quantitative polymerase chain reaction (PCR) kit (Wuhan MSK Biotechnology Co., Ltd., Wuhan, China).

Lentivirus transfection. Equal volumes of miR-29b inhibitor gene lentiviral vector and negative control lentiviral vector were thawed via ice bath, and diluted into $10^{8} \mathrm{TU} / \mathrm{ml}$ with the high-efficiency lentivirus transfection enhancement solution. Rats in the lentivirus group were injected with $150 \mathrm{ml}$ lentivirus solution, and those in the negative lentivirus and control groups were injected with the same amount of the negative control virus solution and high-efficiency lentivirus transfection enhancement solution, respectively.

High-frequency echocardiography. At 3 weeks after the injection of lentivirus, rats were weighed and anesthetized with $10 \%$ chloral hydrate $(200 \mathrm{mg} / \mathrm{kg})$. M-mode echocardiography was performed for all rats using the $7.5 \mathrm{MHz}$ ultrasound diagnostic instrument to detect left ventricular posterior wall thickness (LVPWT), interventricular septum thickness (IVST), left ventricular end-diastolic diameter (LVEDD) and left ventricular end-systolic diameter (LVESD), and left ventricular ejection fraction (LVEF) was calculated using the Teichholtz method.

Treatment of heart specimens of rats. At 3 weeks after transfection (at 6 weeks of feeding), rats were weighed and anesthetized with $10 \%$ chloral hydrate $(200 \mathrm{mg} / \mathrm{kg})$. The chest was opened, and the heart was taken and washed clean. The left ventricle, including interventricular septum, was retained, and left ventricular mass (LVM) (mg) was obtained. LVM index $(\mathrm{LVMI})=\mathrm{LVM} /$ body mass $(\mathrm{BM})$. After the left ventricle was weighed, it was stored in liquid nitrogen used for reverse transcription-quantitative polymerase chain reaction (RT-qPCR).

Detection of miR-29b expression via RT-qPCR. After $50 \mathrm{mg}$ of myocardial tissue was taken and ground in a mortar containing TRIzol reagent, RNA was extracted with chloroform, and the upper aqueous phase was retained and added with 0.5 volume of isopropanol to extract the total RNA. The concentration and purity of RNA extracted were detected using an ultraviolet spectrophotometer (Thermo Fisher Scientific, Inc., Waltham, MA, USA). The absorbance A260/A280 of 1.8-2.0 indicated the qualified purity. The reverse transcription was immediately performed for extracted RNA using the $25 \mu \mathrm{l}$ reaction system prepared according to Table $\mathrm{I}\left(37^{\circ} \mathrm{C}\right.$ for $60 \mathrm{~min}$ and $85^{\circ} \mathrm{C}$ for $5 \mathrm{~min})$. The synthesized complementary deoxyribonucleic acid (cDNA) was diluted by 100 times, and $20 \mu 1$ amplification system was prepared according to Table I, followed by amplification on a PCR instrument in accordance with the procedure in Table II, with U6 as an internal reference (forward primer, 5'-CGCTTCGGC AGCACATATAC-3' and reverse primer, 5'-TTCACGAATT TGCGTGTCAT-3'). miR-29b: forward primer, 5'-ACACT CCAGCTGGGTAGCACCATCTGAAA-3' and reverse primer, 5'-CTCAACTGGTGTCGTGGA-3'. Results were statistically processed using the $2^{-\Delta \Delta \mathrm{Cq}}$ method (14).

Statistical analysis. Cardiac function parameters, blood pressure and PCR results are presented as mean \pm standard 
Table II. PCR procedure.

\begin{tabular}{lcc}
\hline Step & Temperature $\left({ }^{\circ} \mathrm{C}\right)$ & Time \\
\hline Pre-denaturation & 95 & $10 \mathrm{~min}$ \\
$40 \mathrm{Ct}$ & & \\
Denaturation & 95 & $10 \mathrm{sec}$ \\
Annealing & 60 & $20 \mathrm{sec}$ \\
Extension & 72 & $32 \mathrm{sec}$ \\
\hline
\end{tabular}

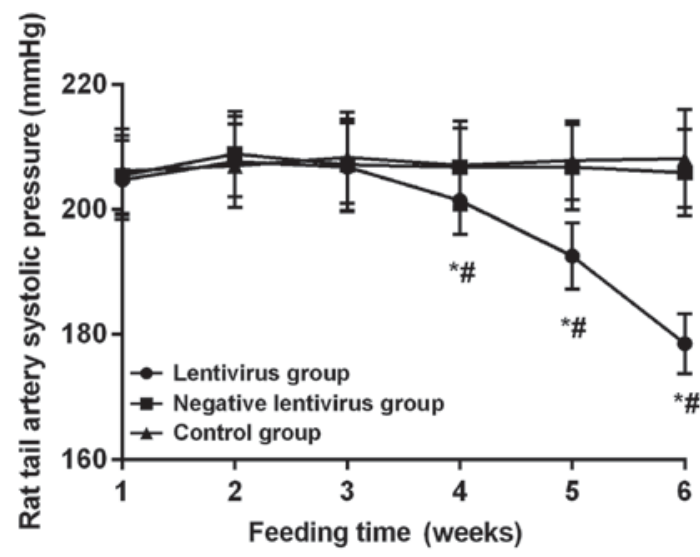

Figure 1. Changes in blood pressure in rats. At 1,2 and 3 weeks after feeding without virus transfection, there was no statistically significant difference in the blood pressure of rats among groups. The blood pressure in the lentivirus group began to drop at 3 weeks after virus transfection, and it was significantly decreased at 4 weeks compared with that at 3 weeks, significantly lower at 6 weeks than that at 5 weeks and also lower at 5 weeks than that 4 weeks, displaying statistically significant differences (all $\mathrm{P}<0.05$ ). There was no statistically significant difference in the blood pressure between the negative lentivirus and control groups during the monitoring for 6 weeks $(\mathrm{P}>0.05)$. The blood pressure in the lentivirus group was obviously lower than that in the negative lentivirus and control groups at 4, 5 and 6 weeks $(\mathrm{P}<0.05) .{ }^{*} \mathrm{P}<0.05$ in comparison with the negative lentivirus and control groups during the same period. ${ }^{*} \mathrm{P}<0.05$ in comparison with the same group in the previous week.

deviation (mean \pm SD). SPSS 20.0 (Asia Analytics, formerly SPSS China) software package was used for data inspection and analysis. Repeated measures analysis of variance was used for the comparison within the same group before and after experiment according to the data distribution characteristics. Analysis of variance was adopted for the comparison among more than three groups with Least Significant Difference test. t-test was used for pairwise comparisons in case of difference. $\mathrm{P}<0.05$ indicates that the difference was statistically significant.

\section{Results}

Blood pressure in rats. At 1, 2 and 3 weeks after feeding without virus transfection, there were no statistically significant differences in the blood pressure of rats among the groups, indicating that the blood pressure is comparable after virus transfection. The blood pressure in the lentivirus group began to drop at 3 weeks after virus transfection, and it was significantly decreased at 4 weeks $(201.43 \pm 5.42 \mathrm{mmHg})$ compared with that at 3 weeks $(206.76 \pm 7.12 \mathrm{mmHg})$ significantly lower at 6 weeks $(178.52 \pm 4.82 \mathrm{mmHg})$ than that at

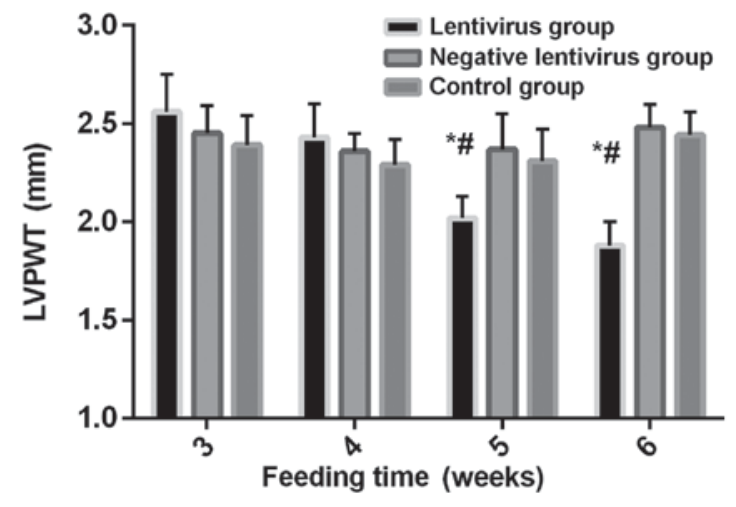

Figure 2. Comparison of LVPWT among groups. Before virus transfection, there was no statistically significant difference in LVPWT among the three groups $(\mathrm{P}>0.05)$. After virus transfection, LVPWT in the lentivirus group showed a decreasing trend, and it was significantly decreased at 5 and 6 weeks compared with that in the previous week, and significantly lower than that in the negative lentivirus and control groups during the same period (all $\mathrm{P}<0.05)$. ${ }^{*} \mathrm{P}<0.05$ in comparison with the negative lentivirus and control groups during the same period. ${ }^{*} \mathrm{P}<0.05$ in comparison with the same group in the previous week.

5 weeks $(192.52 \pm 5.25 \mathrm{mmHg})$ and also lower at 5 weeks than that at 4 weeks, displaying statistically significant differences (all $\mathrm{P}<0.05$ ). The blood pressure declined by $29.13 \mathrm{mmHg}$ on average at 6 weeks after virus transfection compared with that before transfection. There was no statistically significant difference in the blood pressure between the negative lentivirus and control groups during the monitoring for 6 weeks $(\mathrm{P}>0.05)$. The blood pressure in the lentivirus group was obviously lower than that in the negative lentivirus and control groups at 4, 5 and 6 weeks $(\mathrm{P}<0.05)$ (Fig. 1).

Comparison of echocardiographic indexes among the three groups. At 3 weeks without virus transfection, no statistically significant differences were found in LVPWT, IVST, LVEDD, LVESD and LVEF among the three groups $(\mathrm{P}>0.05)$. Before virus transfection, there were no statistically significant differences in LVPWT among the three groups $(\mathrm{P}>0.05)$. After virus transfection, LVPWT in the lentivirus group showed a decreasing trend, and it was significantly decreased at 5 weeks and 6 weeks compared with that in the previous week, and significantly lower than that in the negative lentivirus and control groups during the same period (all $\mathrm{P}<0.05$ ). Before virus transfection, there were no statistically significant differences in LVEDD among the three groups $(\mathrm{P}>0.05)$. After virus transfection, LVEDD in the lentivirus group was gradually increased, and it was obviously higher at 6 weeks than that at 5 weeks, and also higher than that in the negative lentivirus and control groups during the same period. There was no difference between the negative lentivirus and control groups (all $\mathrm{P}<0.05$ ). LVPWT, IVST, LVEDD, LVESD and LVEF had no remarkable differences between the negative lentivirus and control groups and at each time-point within the group (Figs. 2-6).

Comparison of LVMI. At 6 weeks, there was no significant difference in body weight (BW) among the groups, but LVM and LVMI in the lentivirus group were obviously lower than those in the negative lentivirus and control groups $(\mathrm{P}<0.05)$ (Table III). 


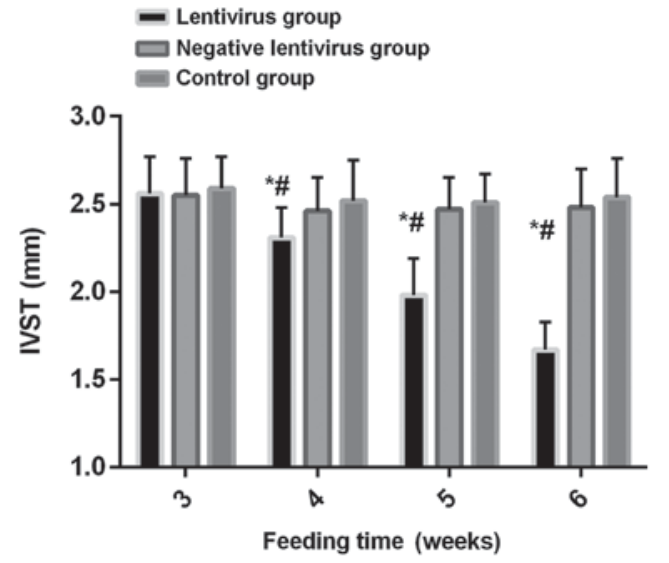

Figure 3. Comparison of IVST among the groups. Before virus transfection, there was no statistically significant difference in IVST among the three groups $(\mathrm{P}>0.05)$. After virus transfection, IVST in the lentivirus group showed a decreasing trend, significantly declined at 4, 5 and 6 weeks compared with that in the previous week, and was obviously lower than those in the negative lentivirus and control groups during the same period. There was no difference between the negative lentivirus and control groups, and no remarkable difference was found at each time-point within the two contro groups $(\mathrm{P}<0.05) .{ }^{*} \mathrm{P}<0.05$ in comparison with the negative lentivirus and control groups during the same period. ${ }^{\text {}} \mathrm{P}<0.05$ in comparision with the same group in the previous week.

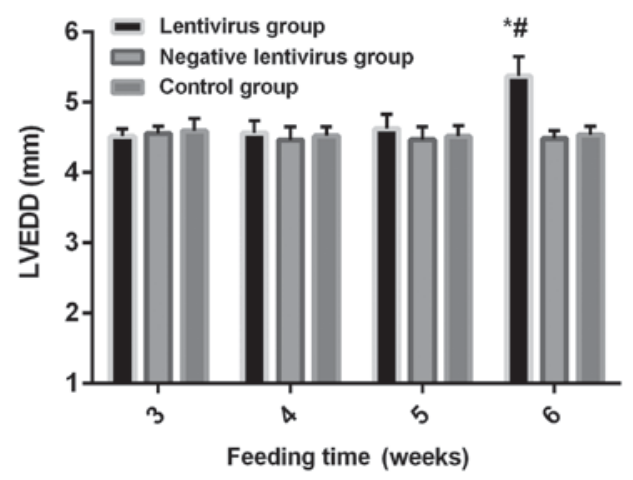

Figure 4. Comparison of LVEDD among the groups. Before virus transfection, there was no statistically significant difference in LVEDD among the three groups $(\mathrm{P}>0.05)$. After virus transfection, LVEDD in the lentivirus group was gradually increased, and it is was obviously higher at 6 weeks than that at 5 weeks, and also higher than that in the negative lentivirus and control groups during the same period. There was no difference between the negative lentivirus and control groups, and no remarkable difference was found at each time-point within the two control groups (all $\mathrm{P}<0.05$ ). ${ }^{*} \mathrm{P}<0.05$ in comparison with the negative lentivirus and control groups during the same period. ${ }^{~} \mathrm{P}<0.05$ in comparison with the same group in the previous week.

Detection of miR-29b expression via RT-qPCR. According to results of RT-qPCR analysis of miR-29b expression in left ventricular tissues of rats, the expression of miR-29b in the lentivirus group was significantly higher than those in the negative lentivirus and control groups $(\mathrm{P}<0.05)$, and it had no statistically significant difference between the negative lentivirus and control groups (Fig. 7).

\section{Discussion}

With the development of medical science in recent years, the diagnostic criteria for hypertension have been constantly improving, the detection rate of hypertension has been

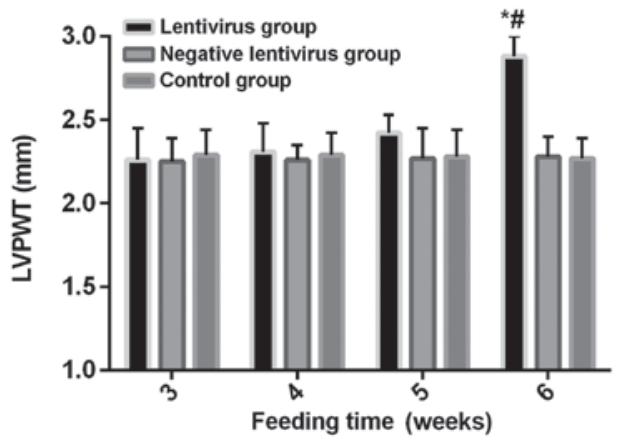

Figure 5. Comparison of LVESD among the groups. Before virus transfection, there was no statistically significant difference in LVESD among the three groups $(\mathrm{P}>0.05)$. After virus transfection, LVESD in the lentivirus group gradually increased, and it was obviously higher at 6 weeks than that at 5 weeks, and also higher than that in the negative lentivirus and control groups during the same period (all $\mathrm{P}<0.05$ ). LVESD in the lentivirus group at 5 weeks was higher than that in the negative lentivirus and control groups during the same period (all $\mathrm{P}<0.05$ ). ${ }^{*} \mathrm{P}<0.05$ in comparison with the negative lentivirus and control groups during the same period. ${ }^{\text {}} \mathrm{P}<0.05$ in comparison with the same group in the previous week.

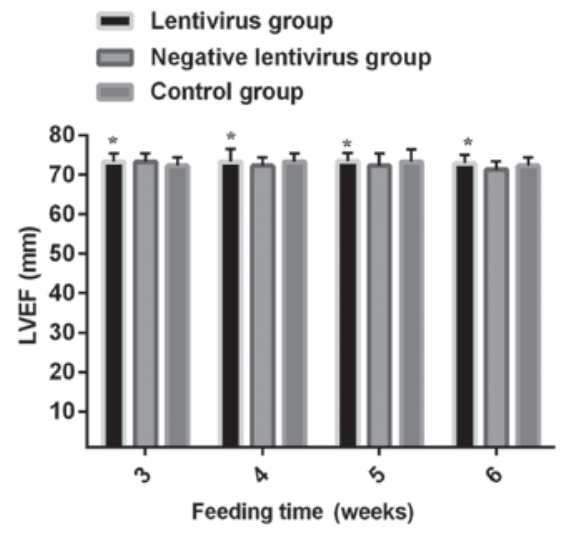

Figure 6. Comparison of LVEF among the groups. Before and after virus transfection, there was no statistically significant difference in LVEF among the three groups of rats $(\mathrm{P}>0.05)$. ${ }^{\text {P }}>0.05$ in comparison with the negative lentivirus and control groups during the same period.

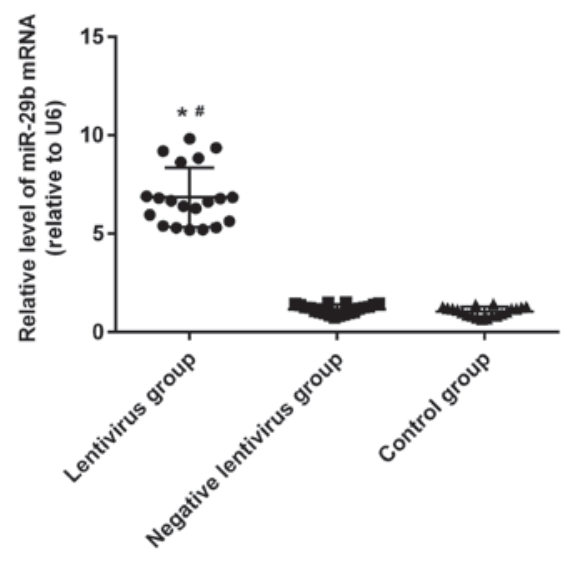

Figure 7. Detection of miR-29b expression via RT-qPCR. According to the results of RT-qPCR analysis of miR-29b expression in the left ventricular tissues of rats, the expression of miR-29b in the lentivirus group was significantly higher than that in the negative lentivirus and control groups $(\mathrm{P}<0.05)$, and it had no statistically significant difference between the negative lentivirus and control groups. " $\mathrm{P}<0.05$ in comparison with the negative lentivirus and control groups during the same period. ${ }^{~} \mathrm{P}<0.05$ in comparison with the control group during the same period. 
Table III. Comparison of LVMI.

\begin{tabular}{lccc}
\hline Groups & LVM $(\mathrm{mg})$ & BW $(\mathrm{g})$ & LVMI $(\mathrm{mg} / \mathrm{g})$ \\
\hline Lentivirus & $720.41 \pm 40.23^{\mathrm{a}}$ & $251.41 \pm 7.17$ & $2.87 \pm 5.61$ \\
Negative lentivirus & $890.92 \pm 88.81$ & $248.51 \pm 7.32$ & $3.59 \pm 12.13$ \\
Control & $910.18 \pm 90.44$ & $253.71 \pm 7.54$ & $3.59 \pm 12.00$ \\
F & 16.66 & 1.13 & 1.46 \\
P-value & $<0.001$ & 0.39 & 0.26 \\
\hline
\end{tabular}

${ }^{\mathrm{a}}<0.05$ in comparison with the negative lentivirus and control groups during the same period.

increasing continuously and the therapeutic regimen has also been continuously perfecting. However, there is still a big gap in China compared with developed countries (15). The prevalence rate of hypertension is still increasing, and it is conservatively estimated that there are at least 200 million people with hypertension in China currently (16). At present, therapeutic drugs for hypertension need to be taken for a long time, there will be a rebound easily after drug withdrawal, the blood pressure can be maintained for a short time, and they have such toxic and side effects as increased serum potassium (17). miR-29b is necessary for normal endothelial function, which can resist fibrosis and cardiac metabolic disturbance (18). Therefore, the mechanism of hypertension in cardiovascular diseases was explored in this study, so as to provide new ideas for primary hypertension and cardiovascular and cerebrovascular diseases to develop new molecular therapy.

It was found in the detection of miR-29b expression in left ventricular tissues of rats via RT-qPCR that the expression of miR-29b in the lentivirus group was significantly higher than that in the negative lentivirus and control groups. Results of this study revealed that there was no significant difference in systolic pressure among groups before transfection, and it began to decline gradually in the lentivirus group after transfection with miR-29b and was always lower than that in the negative lentivirus and control groups, suggesting that miR-29b inhibits the increase in systolic pressure of rats and alleviates hypertension. After transfection with miR-29b, LVPWT and IVST in the lentivirus group displayed decreasing trends, LVEDD and LVESD showed increasing trends, and LVEF remained essentially unchanged. After transfection, BW had no significant difference among groups, but LVM and LVMI in the lentivirus group were remarkably lower than those in the negative lentivirus and control groups, indicating that the overexpression of miR-29b reverses the left ventricular hypertrophy. Liu et al (19) found that Smad3 reverses the downregulation of $\mathrm{miR}-29 \mathrm{~b}$ in renal tissues in hypertensive nephropathy through nuclear factor- $\kappa \mathrm{B}(\mathrm{NF}-\kappa \mathrm{B})$, thus, suppressing hypertensive nephropathy. Zhu et al (20) studied the preventive effect of berberine on cardiovasculzar diseases, and they also found that it relieves cardiac remodeling, promotes angiogenesis and reduces infarct size through significantly increasing the expression level of miR-29b, thus, improving cardiac function. Moreover, the expression of miR-29b also significantly declines in model rats with pulmonary hypertension (13). It was found in this study that the overexpression of miR-29b in the rat model of hypertension could improve cardiac function, indicating that miR-29b possesses the therapeutic potential for hypertension and left ventricular hypertrophy. miR-29b also exerts a protective effect on the heart in ventricular remodeling involving AngII.

The rat model of spontaneous hypertension was used in the present study, and it is more representative for the spontaneous occurrence of hypertension than induced rat model, with a probability of hypertension of $100 \%$ (20). However, there were also shortcomings in this study; the occurrence and development of hypertension lasted for a long time, there were various influencing factors, the mechanism was complex involving a wide range, it was difficult to simulate and restore in vitro, and the rat model of spontaneous hypertension constructed could not fully represent the actual situation. In the subsequent study, therefore, relevant clinical data should be collected, and the correlation between actual expression and hypertension should be analyzed, so as to further verify the conclusion in this study. Besides, it was found in this study that miR-29b could indeed reduce blood pressure and improve cardiac function, but through which target protein it regulates and through which signaling pathway it causes its effects need further experiments. It is suggested that TargetScan and other online software be used to predict the target protein of miR-29b, and western blotting be performed for verification.

In conclusion, the overexpression of miR-29b can improve cardiac function, and inhibiting the miR-29b expression can reduce blood pressure and obviously improve the cardiac function in hypertension rats.

\section{Acknowledgements}

Not applicable.

\section{Funding}

No funding was received.

\section{Availability of data and materials}

The datasets used and/or analyzed during the present study are available from the corresponding author on reasonable request.

\section{Authors' contributions}

$\mathrm{XH}$ and $\mathrm{CW}$ assisted with the design of the rat model. YL and $\mathrm{ZJ}$ performed the PCR. BZ and YD interpreted the 
high-frequency echocardiography result. All authors read and approved the final manuscript.

\section{Ethics approval and consent to participate}

The study was approved by the Ethics Committee of Tianjin Hospital of ITCWM, Nankai Hospital (Nankai, China).

\section{Patient consent for publication}

Not applicable.

\section{Competing interests}

The authors declare that they have no competing interests.

\section{References}

1. Li P, Guo W, Du L, Zhao J, Wang Y, Liu L, Hu Y and Hou Y microRNA-29b contributes to pre-eclampsia through its effects on apoptosis, invasion and angiogenesis of trophoblast cells. Clin Sci (Lond) 124: 27-40, 2013.

2. Takase S, Lerond L, Bergan JJ and Schmid-Schönbein GW: The inflammatory reaction during venous hypertension in the rat. Microcirculation 7: 41-52, 2000.

3. Volmink J, Bradley H, Maroney R, Maroney R, Mbewu A, Opie LH and Volmink J: Betablockers for hypertension. Cochrane Database Syst Rev 1: CD002003, 2007.

4. Liu JQ, Zelko IN, Erbynn EM, Sham JS and Folz RJ: Hypoxic pulmonary hypertension: Role of superoxide and NADPH oxidase (gp91phox). Am J Physiol Lung Cell Mol Physiol 290: L2-L10, 2006.

5. Sung YK and Chung L: Connective tissue disease-associated pulmonary arterial hypertension. Rheum Dis Clin North Am 41: 295-313, 2015.

6. Rich S and Rabinovitch M: Diagnosis and treatment of secondary (non-category 1) pulmonary hypertension. Circulation 118: 2190-2199, 2008.

7. Scherrer JF, Xian H, Bucholz KK, Eisen SA, Lyons MJ, Goldberg J, Tsuang M and True WR: A twin study of depression symptoms, hypertension, and heart disease in middle-aged men. Psychosom Med 65: 548-557, 2003.

8. Yan B, Guo Q, Fu FJ, Wang Z, Yin Z, Wei YB and Yang JR: The role of miR-29b in cancer: Regulation, function, and signaling. Onco Targets Ther 8: 539-548, 2015.

9. Chaturvedi P, Kalani A, Medina I, Familtseva A and Tyagi SC: Cardiosome mediated regulation of MMP9 in diabetic heart: Role of mir29b and mir455 in exercise. J Cell Mol Med 19: 2153-2161, 2015
10. Zhang Y, Huang XR, Wei LH, Chung AC, Yu CM and Lan HY: miR-29b as a therapeutic agent for angiotensin II-induced cardiac fibrosis by targeting TGF- $\beta / \mathrm{Smad} 3$ signaling. Mol Ther 22 : 974-985, 2014.

11. Widlansky ME, Jensen DM, Wang J, Liu Y, Geurts AM, Kriegel AJ, Liu P, Ying R, Zhang G, Casati M, et al: miR-29 contributes to normal endothelial function and can restore it in cardiometabolic disorders. EMBO Mol Med 10: e8046, 2018.

12. Luo Y, Dong HY, Zhang B, Feng Z, Liu Y, Gao YQ, Dong MQ and Li ZC: miR-29a-3p attenuates hypoxic pulmonary hypertension by inhibiting pulmonary adventitial fibroblast activation. Hypertension 65: 414-420, 2015.

13. Chen J, Li Y, Li Y, Xie L, Wang J, Zhang Y and Xiao T: Effect of miR-29b on the proliferation and apoptosis of pulmonary artery smooth muscle cells by targeting Mcl-1 and CCND2. Biomed Res Int 2018: 6051407, 2018.

14. Livak KJ and Schmittgen TD: Analysis of relative gene expression data using real-time quantitative PCR and the 2(-Delta Delta C(T)) method. Methods 25: 402-408, 2001.

15. Coghlan JG, Denton CP, Grünig E, Bonderman D, Distler O, Khanna D, Müller-Ladner U, Pope JE, Vonk MC, Doelberg M, et al; DETECT study group: Evidence-based detection of pulmonary arterial hypertension in systemic sclerosis: The DETECT study. Ann Rheum Dis 73: 1340-1349, 2014.

16. Rosenkranz S, Gibbs JS, Wachter R, De Marco T, VonkNoordegraaf A and Vachiéry JL: Left ventricular heart failure and pulmonary hypertension. Eur Heart J 37: 942-954, 2016.

17. Taichman DB, Ornelas J, Chung L, Klinger JR, Lewis S Mandel J, Palevsky HI, Rich S, Sood N, Rosenzweig EB, et al: Pharmacologic therapy for pulmonary arterial hypertension in adults: CHEST guideline and expert panel report. Chest 146: 449-475, 2014.

18. Steele R, Mott JL and Ray RB: MBP-1 upregulates miR-29b that represses Mcl-1, collagens, and matrix-metalloproteinase- 2 in prostate cancer cells. Genes Cancer 1: 381-387, 2010.

19. Liu GX, Li YQ, Huang XR, Wei LH, Zhang Y, Feng M, Meng XM, Chen HY, Shi YJ and Lan HY: Smad7 inhibits AngII-mediated hypertensive nephropathy in a mouse model of hypertension. Clin Sci (Lond) 127: 195-208, 2014.

20. Zhu ML, Yin YL, Ping S, Yu HY, Wan GR, Jian X and Li P: Berberine promotes ischemia-induced angiogenesis in mice heart via upregulation of microRNA-29b. Clin Exp Hypertens 39: 672-679, 2017.

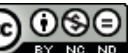

This work is licensed under a Creative Commons Attribution-NonCommercial-NoDerivatives 4.0 International (CC BY-NC-ND 4.0) License. 